\title{
INFORMATION TOURISM SYSTEM WITH MIXED SEMIOTIC STRUCTURE
}

DOI: http://dx.doi.org/10.18509/GBP.2016.19

UDC: 338.48:[007:004

\author{
Eugene Eremchenko ${ }^{1}$, \\ Vladimir Tikunov ${ }^{1}$, \\ Svemir Gorin ${ }^{2}$, \\ Bashkim Idrizi ${ }^{3}$ \\ Ivan Radevski ${ }^{2}$ \\ ${ }^{1}$ Moscow State University, Russia \\ ${ }^{2}$ SS. Cyril and Methodius University, Republic of Macedonia \\ ${ }^{3}$ State University of Tetova, Republic of Macedonia
}

\begin{abstract}
Tourism plays an important role in ensuring the sustainable development of countries and nations in our days, but the management of tourism requires the establishment of specific information products aimed to deliver precise, complete and context information to different population groups. Solving this problem requires the joint use of various semiotics.

We propose to discuss the concept of heterogeneous information system, based on mixed semiotic structure. System consists of to main modes: text, and visual (signless) representation. User can select most useful semiotic model interactively for best efficiency. From this point of view context represented by signless images of different types: satellite imagery, geolocated photos, and spherical panoramas.
\end{abstract}

Keywords: visualization, situational awareness, semiotics, sign, satellite images

\section{INTRODUCTION}

It is widely believed that visual representation is the most efficient way to exchange information. Therefore, visualization is widely used in information systems and, especially, in decision support systems. Tourist information system is a kind of decision support systems, because it aimed to help users in making decisions.

Visualization, or scientific visualization is a significant component of efforts intended to ensure situational awareness in effective decision-making information systems. Scientific visualization is an interdisciplinary approach that «primarily concerned with the visualization of 3-D+ phenomena (architectural, meteorological, medical, biological, etc.), where the emphasis is on realistic renderings of volumes, surfaces, illumination sources, and so forth, perhaps with a dynamic (time) component» [1].

From our point of view this definition describes the phenomenon of scientific visualization correctly [2], but it is not a truly intentional definition. For example, we can not use this definition for correct discrimination scientific visualization from the all possible ways of perception of information. It is strange, but comprehensive classification of different ways of perception of information is still absent, and scientific visualization realm iis not localized yet within variety of different ways for providing information for users.

Implementation of scientific visualization in geospatial applications requires the development of approach to integration of different types of data and different ways of 
information perception. Therefore, as the first step we should provide classification of different ways for information perception. From our point of view, basic classification principle could be provided with the help of semiotics. We intend to consider this type of data as an unsigned, although the debate about the connection between the images and the iconic characters is carried out [3].

There are at least two different approaches for perception: 1) signs, and 2) direct visual perception by the way of unprocessed images. Scientific visualization is a specific approach based on using of direct or (mostly) simulated images for representation of quantitative information.

Article I. Contrariwise, alternative approach is based on using of symbolic representation of data: texts, mathematical symbols, mapping signs, etc. So we can divide the landscape of perception into two detached realms: 1) perception through the signs, and, 2) perception through the direct images (Figure 1). Signs mean letters, symbols, mapping signs, iconic images, etc. Direct perception includes visual phenomena like images on retina, photographic images, spherical panoramas, advanced scientific visualization, etc.

\section{DISCUSSION}

The proposed division should be discussed within the semiotic context, because in classical semiotics there are no distinct separation between images as signs (so called «iconic images») and direct visual perception [4]. In semiotics iconic images usually treat as most simple signs, because iconic images resembles visual appearance of signified. The border between the image that resembling visual appearance of signified and its visual appearance itself is blurred. As a rule iconic signs is a pictures - images created or processed by human intentionally. In this paper we propose to separate iconic images (like pictures) and direct visual perception (like remote sensing data, photographic images, spherical panoramas, etc.). This division is discussable too. We plan to discuss the problem of correct separation of iconic images and direct visual perception in future articles.

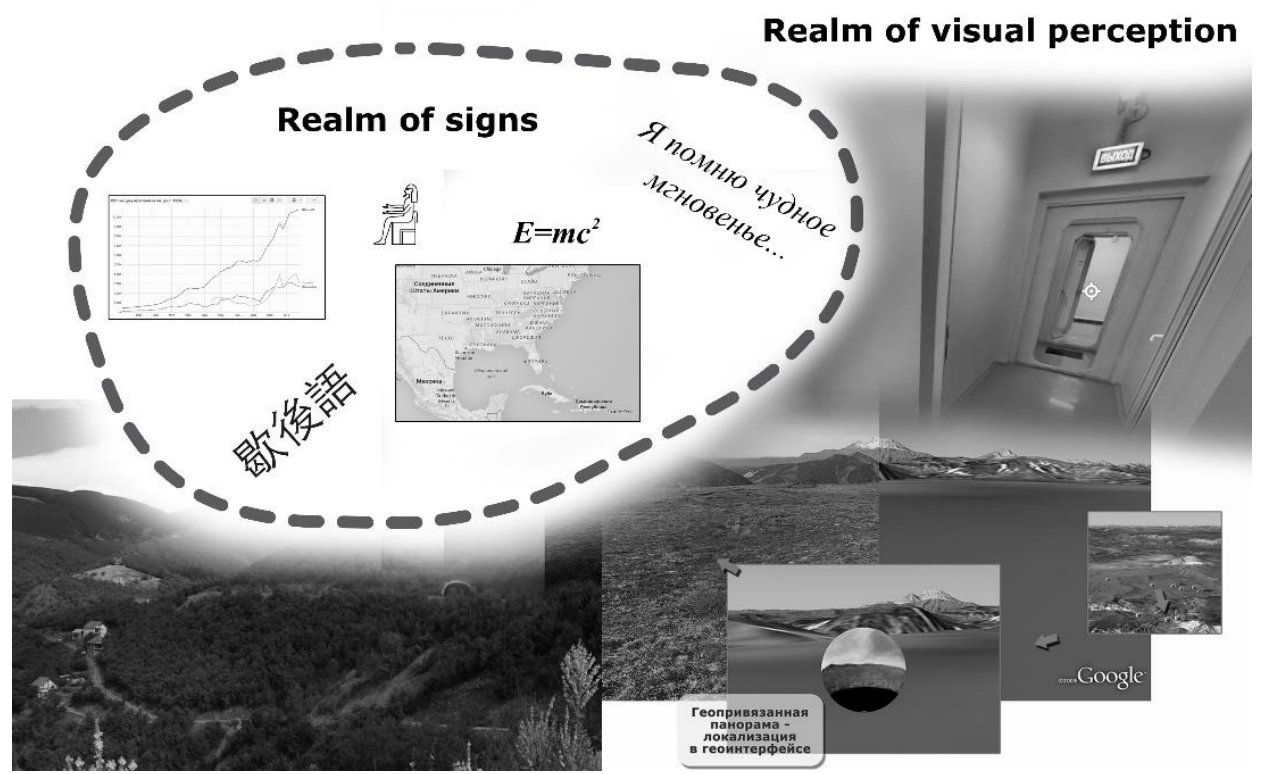

Figure 1. Representation of two realms of approaches for perception from semiotics point of view. 
From the proposed point of view scientific visualization is an attempt to provide artificial visualization that resemble real or virtual objects in a way close to direct «natural» visual perception. So we can select at least three ways of perception:

1. signs;

2. direct visualization;

3. scientific visualization.

Here scientific visualization is a part of the direct perception through visualization by the means of images (Figure 2).

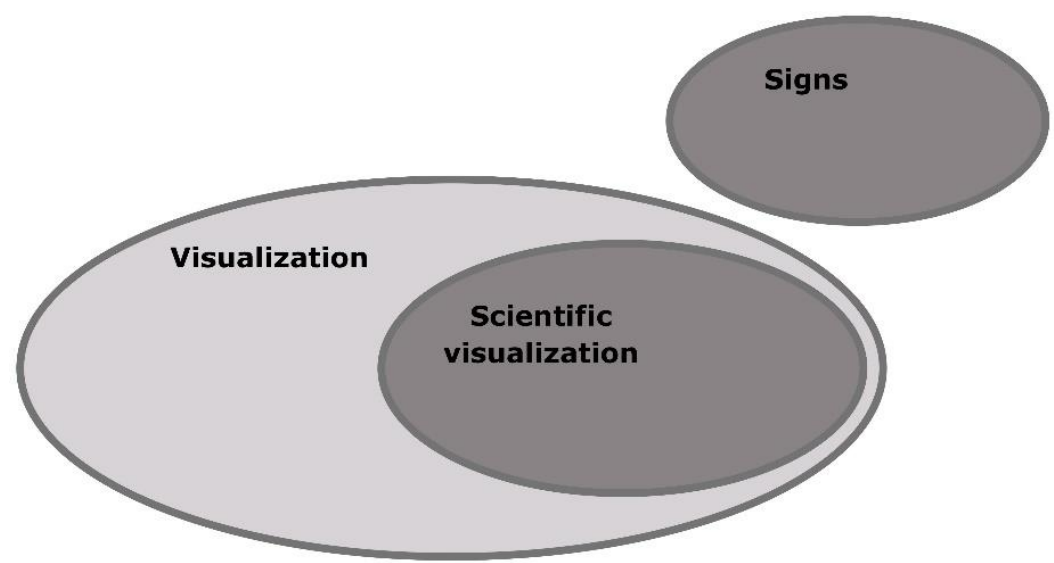

Figure 2. Different realms of perception.

What is most effective way for perception - signs or direct perception? High effectiveness of direct perception and a scientific visualization is a fact. Nevertheless, we can not specify the optimal way for perception, because signs and direct perception describes different aspects of situation. Generally direct perception (and images at all) provide understanding of spatial relations within the environment, and signs provide understanding of temporal evolution of the elements of the environment. Information system should provides different ways for perception - signs, images, rich scientific visualization by the means of multi- and hypermedia, etc. Architecture of information system should provide equitable access for different ways for perception of whole environment for users.

More importantly, heterogeneous dataset with different ways for perception should provide tbe best possible level of situational awareness for users. Situational awareness is defined as a principle of "perception of elements in the environment within a volume of time and space" [5], so the universal environment model is sine qua non requirement of the situational awareness. But mapping representation is scale-dependent; existence of universal map, for all possible scales is directly prohibited by the rules of cartography. Raster images is the only possible way for providing spatial framework that could support situational awareness. So the semiotic model based on signless representation of spatial framework and sign representation of elements within this framework was proposed (Figure 3). Visual images is a best way for perception of spatial framework; on the other hand signs is a best way for providing access to temporal information (semantics, descriptions, etc.). 


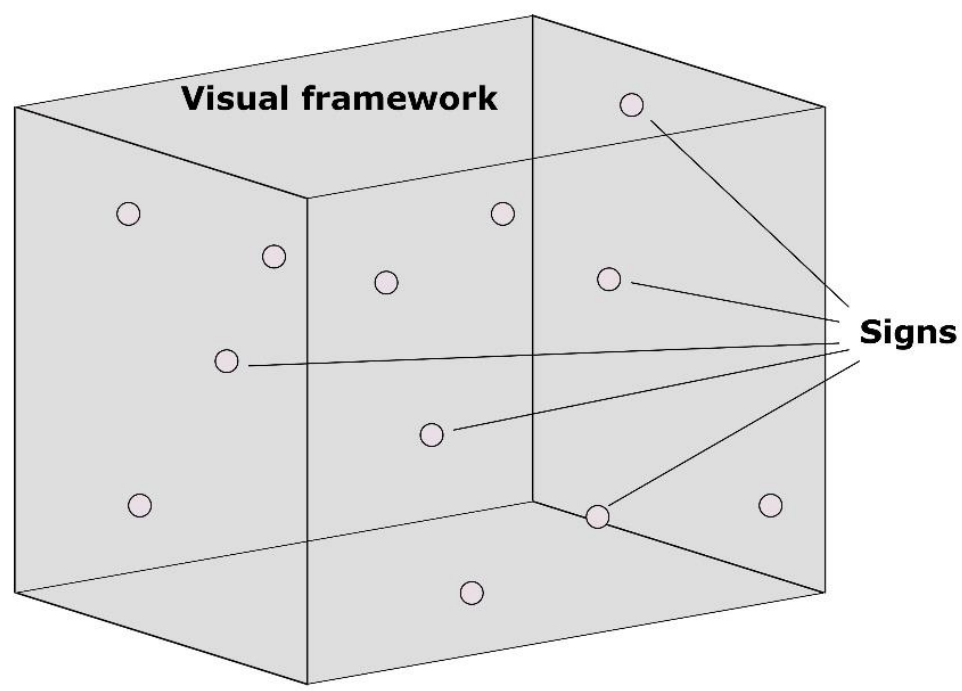

Figure 3. Simplified semiotic vision of structure of information system.

\section{IMPLEMENTATION OF THE APPROACH TO GEOVISUALIZATION}

The proposed vision of the structure of the information system was implemented in the concept of universal information system designed primarily for tourist and educational purposes and could be extended on different touristic regions and activities [6]. The main purpose in this case was to provide an acceptable level of situational awareness for users. Systems includes two basic elements: 1) textual, and 2) visual (signless) representation with hypertext links between both of them. User can change modes interactively.

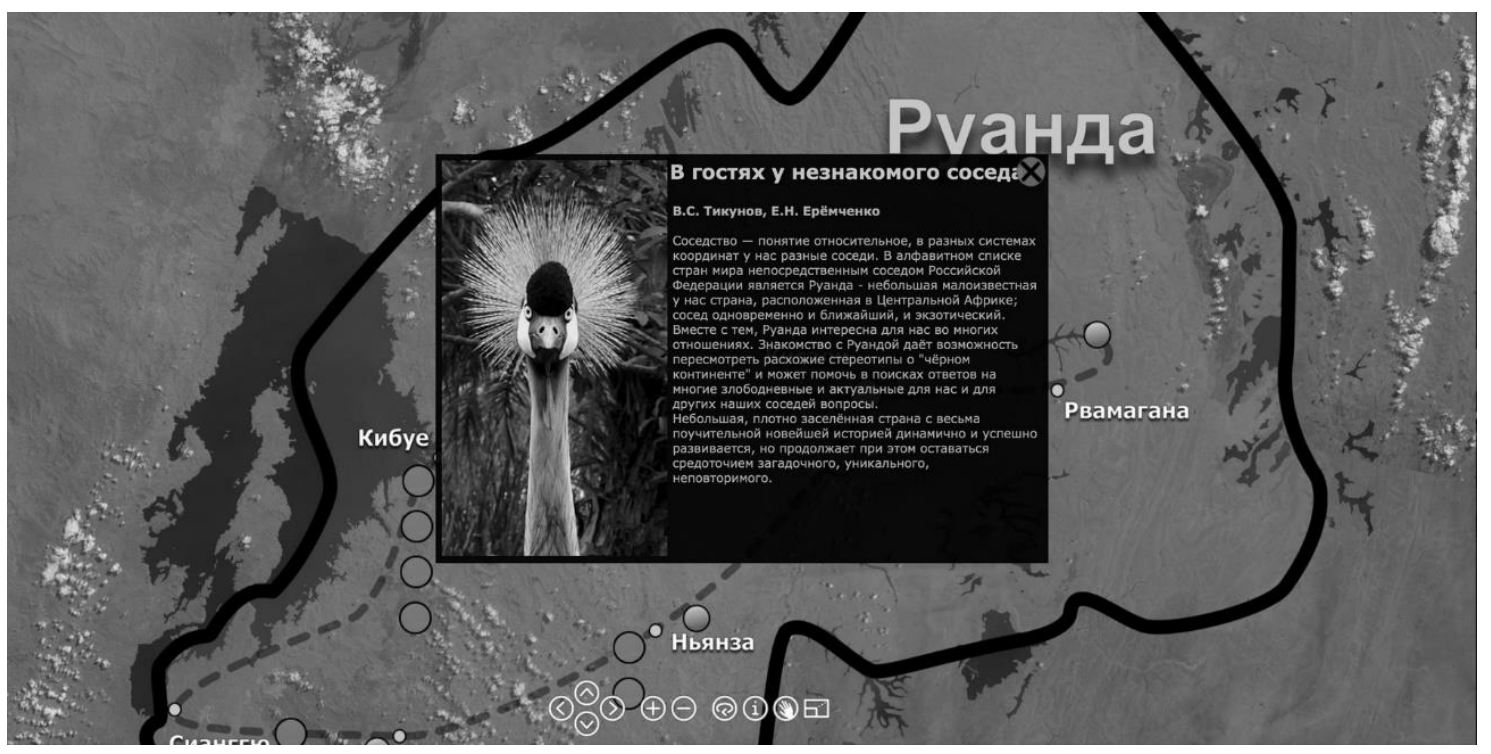

Figure 4. User interface of information system of Rwanda.

Up to now two prototypes of the system were established: 1) information system for Rwanda, based on data collected in 2014, and 2) information system for South East Balkans region, based on data collected in 2015. Both systems were created as web applications supported by wide range of present browsers (Mozilla Firefox, Google Chrome, Internet Explorer, Safari, Opera). Systems based on Adobe Flash multimedia 
platform and created with the help of Pano2VR application. Currently, both systems support the Russian language only, but it is planned to provide multi-lingual interface.

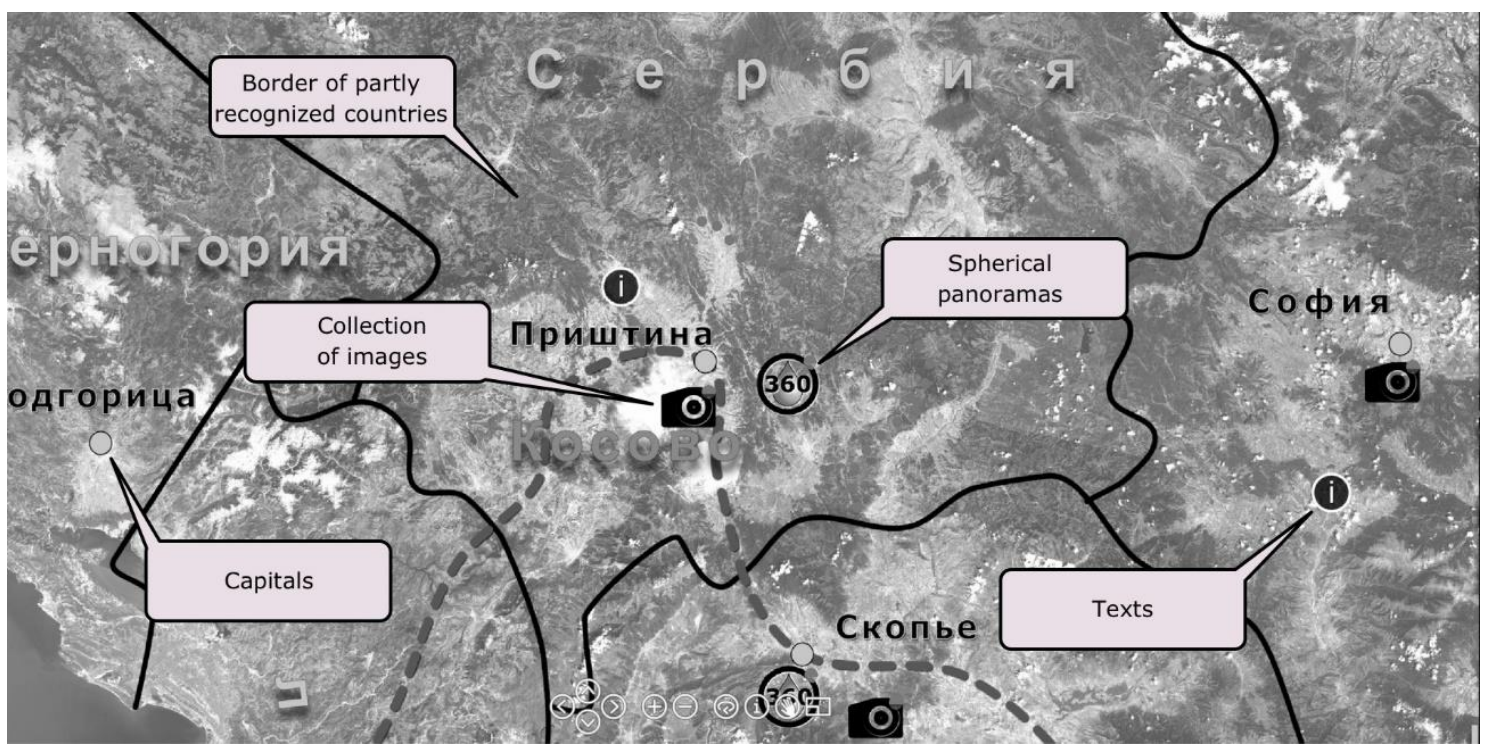

Figure 5. Anchor points and elements of user interface of prototype of South Balkans information system.

Systems includes different types of data: texts, ordinary images, satellite images, pictures for the visual representation of quantitative indicators, spherical panoramas. User can enter the system from the different points of entrance: for example, through the texts or images.

The basic spatial framework is defined by raster mosaic coverage, created from LandSat true color satellite images, because spatial resolution of this remote sensing data (near 15 $\mathrm{m}$ per pixel) is a most suitable choice for the intended purposes of the system in all relevant scales. More higher resolution leads to very big volumes of data; more lesser resolution does not provide appropriate level of user experience. Coverage could be zoomed in or out interactively for the best resolution/area ratio. Raster mosaic is combined with rasterized and generalized non-interactive vector mapping data - state borders, routes, capitals and towns, points of interest, toponyms.
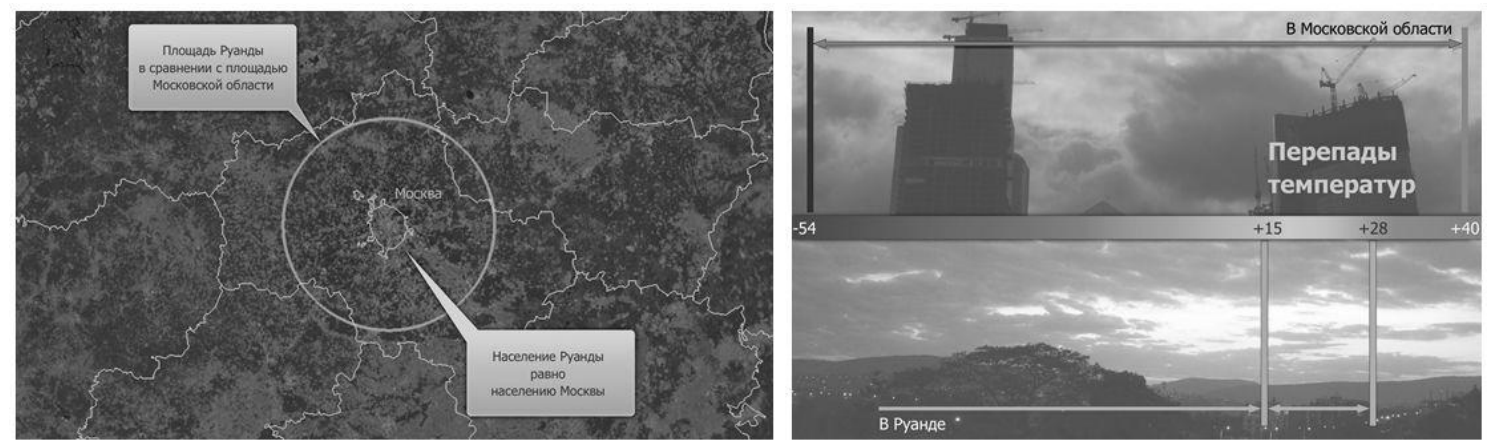

Figure 6 a, b. Visual comparative representation of a quantitative parameters . Left to right: 6a - The area and the population of Rwanda in comparison with the area and the population of Moscow region, Russia.

Area of Rwanda represented by the circle overlapped border of Moscow region. $6 \mathrm{~b}$ - maximum and minimum temperatures of Rwanda in comparison with the same parameters of Moscow region. 
Visual components of the systems are represented by the high resolution images and panoramas. All images and panoramas are provide suitable level of detailing. For example, angle resolution of panoramas is about 1 angular minute. Images are created with the help of wide angle lens that provide additional context information for objects of interest. Interactivity of the system is provided by anchor points (fixed to the geospatial context) and control button (fixed to the screen) in the panoramas, as well as hypertext links.

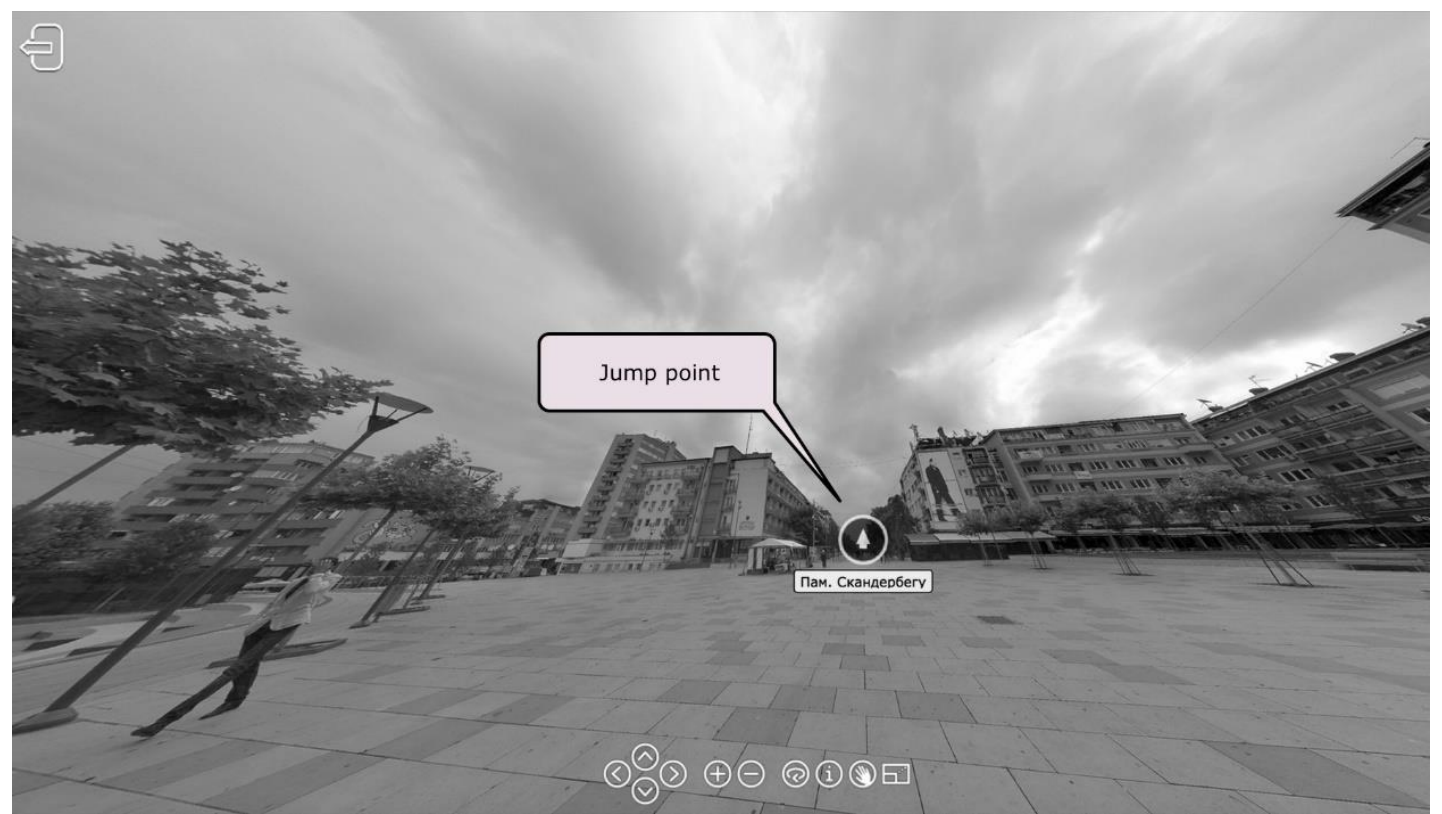

Figure 7. Panorama of Prishtina (wide angle view) with anchor point for jump to next panorama.

Significant problem is providing of situational awareness for quantitative parameters. In described systems visual representation of quantitative parameters were provided (Figure $6 \mathrm{a}, \mathrm{b})$. Images, descriptive texts and pictures are embed into spatial framework. User can access appropriate information clicking anchor points. Also user can enter the system via text or image collections. It means in any cases all possible content is accessible. Content is provided with context (both visual and textual) that assuring situational awareness.

Images, descriptive texts and pictures are embed into spatial framework. User can access appropriate information clicking anchor point (Figure7). Also user can enter the system via text or image collections. It means in any cases all possible content is accessible. Content is provided with context (both visual and textual) that assuring situational awareness.

\section{CONCLUSIONS}

The proposed concept of the tourism destination information system designed to study the best possible ways to provide situational awareness in heterogeneous data sets from the semiotic point of view. The concept behind the tourist information products of this kind can be described as "minimize the signs".

In present the functionality of both existed models are limited; we plan to improve models. First of all, we plan to provide dynamics and 3D-representation of spatial 
framework, rich visual and textual context, multi-lingual support. It seems very promising to use in touristic applications anamorphic images [7].

Assessment of situational awareness is a significant problem, because there are no widely accepted methodologies for measuring of shares of elements with different semiotics in model and for measuring of situational awareness. There are some ideas about methodology for estimating the shares of different semitotic elements in heterogeneous atlases [4]. Nevertheless, the problem of measuring of situational awareness is still unsolved yet and should be solved in future.

\section{ACKNOWLEDGEMENT}

The study was supported by grant of the Russian Science Foundation (project №15-170009).

\section{REFERENCES}

[1] Friedly, M. Milestones in the history of thematic cartography, statistical graphics, and data visualization, 2009, [Online] http://www.math.yorku.ca/SCS/Gallery/milestone/milestone.pdf (Revised: 15.12.2015)

[2] Rosenblum L, Earnshaw RA, Encarnacao J, Hagen H, Kaufman A, Klimenko S, Nielson G, Post F, Thalmann D, Scientific visualization, VRLAB-BOOK-2007-014, Academic Press, 1994

[3] Eremchenko, E., Molodan, G., Semichastny, I, Sirenko, V., Wolodtschenko, A. Berichte zu den Seminaren „Neo-Geographie und Metakartosemiotik“, Meta-CartoSemiotics v.6, 2013, [Online] http://meta-cartosemiotics.org/uploads/mcs_vol6_2013/MCS_Vol6_2013_Eremchenko.pdf (Revised: 15.12.2015)

[4] Endsley, Mica, Toward a theory of situation awareness in dynamic systems, Human Factors, vol. 37, no. 1, pp. 32-64, 1995

[5] Wolodtschenko, Alexander, Atlaskartosemiotik: neue Möglichkeiten und Lösungen, Meta-Carto-Semiotics, Vol. 3, [Online] http://meta-cartosemiotics.org/uploads/mcs_vol3_2010/MCS_Vol3_2010_Wolodtschenko.pdf (Revised: 15.12.2015)

[6] Tikunova I.N. The main traits of world tourism geography, Proceeding of International Conference InterCarto/InterGIS-15, v. 2, pp. 654-6615, 2009.

[7] Aleksandrova A.Yu., Tikunova I.N. Application of anamorphic images in tourism geography research. - Tourism Today, N 11, pp. 154-171, 2011. 